\title{
High Prevalence of Vancomycin Non-Susceptible and Multi-Drug Resistant Enterococci in Farmed Animals and Fresh Retail Meats in Bangladesh
}

\author{
Mohammed A. Samad ( $\nabla$ msamad@blri.gov.bd) \\ Bangladesh Livestock Research Institute \\ Md Shahjalal Sagor \\ Bangladesh Livestock Research Institute \\ Muhammad Sazzad Hossain \\ Bangladesh Livestock Research Institute \\ Md Rezaul Karim \\ Bangladesh Livestock Research Institute \\ Mohammad Asheak Mahmud \\ Bangladesh Livestock Research Institute \\ Fahria A. Shownaw \\ Bangladesh Livestock Research Institute \\ Zakaria Mia \\ Jagannath University \\ Md Samun Sarker \\ Bangladesh Livestock Research Institute \\ Roderick M. Card \\ Animal and Plant Health Agency \\ Agnes Agunos \\ Public Health Agency of Canada \\ Lindahl Johanna \\ International Livestock Research Institute
}

\section{Research Article}

Keywords: Enterococcus spp., food safety, livestock, multi-drug resistance, virulence, antimicrobial resistance

Posted Date: November 10th, 2021

DOI: https://doi.org/10.21203/rs.3.rs-1041994/v1

License: (a) (7) This work is licensed under a Creative Commons Attribution 4.0 International License. Read Full License

Version of Record: A version of this preprint was published at Veterinary Research Communications on March 26th, 2022. See the published version at https://doi.org/10.1007/s11259-022-09906-7. 


\section{Abstract}

The emergence of antimicrobial resistant Enterococcus spp., a leading cause of untreatable nosocomial infection, in food animals and dissemination to humans is a public health concern. The study was conducted to determine the prevalence and antimicrobial resistance, and virulence characteristics of Enterococcus faecalis and Enterococcus faecium in food animals and meats in Bangladesh. Enterococcus spp., were confirmed using sodA gene specific PCR, and antimicrobial resistance and virulence properties were characterized by PCR. Enterococcus spp. were recovered from $57 \%$ of the collected samples $(\mathrm{n}=201 / 352)$. Farm samples yielded significantly higher $(\mathrm{p} \leq 0.05)$ prevalence $(62 \%)$ compared to retail meat samples $(41 \%)$ and $E$. faecalis $(52 \%)$ was most frequently isolated species. High proportions of isolates exhibited resistance to tetracycline (74\%), erythromycin (65\%) and ciprofloxacin (34\%). Fifty-one isolates were vancomycin non-susceptible enterococci (VNSE), of which forty-seven were MDR and 20 were linezolid resistant, a last line drug for VNSE. Virulence factors such as gelatinase ( $g$ e/E), aggregation factor (asa1) and sex pheromone ( $c p d)$ were detected along with vancomycin resistance gene (vanA, vanB and vanC2/C3) in VNSE isolates. The high prevalence of MDR enterococci in food animals and retail meats may lead to infection in consumers with concomitant reduced therapeutic options available for treatment.

\section{Introduction}

Enterococci are Gram-positive bacteria belonging to the lactic acid bacterial group which consists of over 50 diverse species, and inhabit the gastrointestinal flora of humans and a wide variety of warm-blooded animals as well as insects (Santestevan et al. 2015). Over the past few decades, Enterococcus spp., particularly Enterococcus faecium and Enterococcus faecalis, have emerged as one of the most challenging healthcare associated pathogens, and is one of the three most common causes of nosocomial infections worldwide (Wang et al. 2015). The spectrum of enterococci-caused infections in humans includes urinary tract infections, peritonitis, endocarditis, neonatal sepsis or even life-threatening infections such as septicemia and meningitis, and with about $80-90 \%$ of all enterococcal clinical infections being caused by E. faecalis and E. faecium (Arias and Murray 2012). Additionally, enterococci are often used as indicators of fecal contamination in food and water, and are able to compete and survive in harsh environments (Lebreton et al. 2014).

Enterococci have low susceptibility to beta-lactams and intrinsic resistance to several antimicrobial classes including sulphonamides, cephalosporins, and low concentrations of aminoglycosides. Indeed, enterococci are able to acquire and exchange genetic elements, including AMR genes, and thereby serve as reservoirs of transferable resistance circulating in Gram positive bacteria (Hollenbeck and Rice 2012; Wang et al. 2015). Acquisition of resistance to antimicrobials such as vancomycin and linezolid in enterococci is of considerable clinical concern, and is the development of multi-drug resistance (MDR) which could restrict treatment options. Vancomycin resistance is conferred by van genes such as vanA, vanB, vanC1, vanC2/C3, and vanD (Kolár 2018). Epidemiological studies have described the prevalence of MDR enterococci in different sources including human, animal, food of animal origin and even in environmental sources such as soil and surface water (Bennani et al. 2012; Cassenego et al. 2011; López-Salas et al. 2013; Torres et al. 2018). The emergence and rapid spread of MDR poses a serious therapeutic challenge for effective antimicrobial therapy in human infections, due to scarcity of newer antimicrobial agents as well as fewer remaining therapeutic options caused by Gram positive organisms (Aslam et al. 2012; Wang et al. 2015). An additional concern is the presence of virulence genes and diverse virulence factors have been reported in enterococci that increase their ability to colonize hospitalized patients and contribute to infections in humans (Mannu et al. 2003).

Antimicrobial resistant enterococci have been observed in food animals and food of animal origin, such as poultry, duck, swine and cattle (Vignaroli et al. 2011), which is an indication of fecal contamination; meat products are therefore at risk of becoming contaminated during the slaughtering process (Boehm and Sassoubre 2014; Tyson et al. 2018; Wang et al. 2015). The literature suggests food animals as reservoir of antimicrobial resistant enterococci, and contaminated products could enter the food chain (Aslam et al. 2012; Hammerum et al. 2010; Hoelzer et al. 2017; Ogier and Serror 2008) in the absence or lack of strict food safety measures in place. Moreover, enterococci from farm animals and food of animal origin can transfer their resistance genes, located on mobile genetic elements, to human endogenous flora as well as transient bacteria, including pathogens (Vignaroli et al. 2011).

The irrational use of antimicrobials with growth promotion properties in livestock and poultry has been identified as a risk factor for the emergence of antimicrobial resistant Enterococcus spp (Hoelzer et al. 2017). In Bangladesh, there are over 250 antibiotics registered for human and 100 antibiotics registered for veterinary medicine use according to the Directorate General of Drug Administration (DGDA), Bangladesh database (DGDB 2018). However, vast majority of the veterinary medicinal products registered are categorized by the World Health Organization as critically important antibiotics (CIAs) in human medicine (World Health Organisation 2019)

In Bangladesh, the prevalence and diversity of enterococci such as E. faecalis and E. faecium in livestock, poultry and in animal origin food products is poorly defined. Furthermore, little is known about the antibiotic resistances and virulence genes harboured in these bacteria. To address this evidence gap, we have undertaken this study to determine the prevalence of enterococci in farm animals and retail meat, and explored their AMR patterns. E. faecalis and E. faecium were isolated and tested for susceptibility using a panel of eight antimicrobials important in human and veterinary medicine, and vancomycin resistant isolates were further screened for the presence of virulence genes.

\section{Materials And Methods}

\section{Sample collection and isolation of Enterococcus spp.}

A total of 352 samples were collected from selected farms and at retail markets of Savar, Gazipur, and Dhaka from 2016 to 2017 . The sampling frame for the farm component constituted poultry $(n=136)$, cattle $(n=35)$, goat $(n=29)$ and camel $(n=30)$. Cloacal swabs were collected from poultry using sterile swab, and for cattle, goat, and camel samples approximately $5 \mathrm{~g}$ of freshly excreted fecal contents was collected into $25 \mathrm{ml}$ PBS. For meat products, approximately $5 \mathrm{~g}$ of chicken $(n=60)$, beef $(n=32)$ and mutton $(n=30)$ were collected aseptically into $10 \mathrm{ml}$ PBS, and stored on ice for transport to the laboratory within 3-5 hours. All samples were processed using routine bacterial culture for enterococci as per standard protocols (Kuiken et al. 2005) and as outlined below. In brief, 
samples (cloacal swab or $1 \mathrm{ml}$ of homogenized meat) were cultured in selective pre-enrichment broth (brain heart infusion broth [BHI]; BD Difco ${ }^{\mathrm{TM}}$, USA) supplemented with $5 \% \mathrm{NaCl}$ at $37^{\circ} \mathrm{C}$ for $24-48$ hours. Primary isolation of enterococci was done using kanamycin aesculin azide (KAA) agar (Oxoid, UK) at $37^{\circ} \mathrm{C}$ for $18-24$ hours and further screening was done by transferring 3-5 characteristic brown colonies from KAA agar to $5 \%$ sheep blood agar (Oxoid, UK) for another 24 hours at $37^{\circ} \mathrm{C}$, followed by biochemical and temperature tolerance test (i.e. $10^{\circ} \mathrm{C}$ then to $40^{\circ} \mathrm{C}$ ) (Guerrero-Ramos et al. 2016). Final confirmation of species identification was done for one or two selected pure isolates for each primary positive sample, using u-PCR I and $\mathrm{m}$-PCR I based on enterococci specific sodA gene. Genus specific primer for Enterococcus spp., and species-specific primer for E. faecalis and E. faecium were used for molecular confirmation of enterococci isolates according to previously published protocol (Jackson et al. 2004; Poyart et al. 2000) with a slight modification. DNA extraction was performed using conventional boiling method (Huq et al. 2012). List of primer and amplified target DNA size used in this study are provided in Supplementary material 1.

\section{Antimicrobial susceptibility assay}

Kirby-Bauer disk diffusion assay was performed according to Clinical and Laboratory Standards Institute standards M02-A12 and M07-A10 (Tuohy et al. 2000). Quality control strain E. faecalis ATCC 29212 was used as control for antimicrobial susceptibility testing. E. faecalis and E. faecium isolates were susceptibility tested using a panel of eight antimicrobials commonly used in veterinary and human medicine (Oxoid, Hampshire, UK) belong to phenicols (chloramphenicol: $30 \mu \mathrm{g}$ ), quinolones (ciprofloxacin: $5 \mu \mathrm{g}$ ), macrolides (erythromycin: $15 \mu \mathrm{g}$ ), oxazolidinones (linezolid: $30 \mu \mathrm{g})$, glycopeptides (vancomycin: $30 \mu \mathrm{g}$ ), penicillins (penicillin G: $10 \mu \mathrm{g}$ ), nitrofurans (nitrofurantoin: $300 \mu \mathrm{g}$ ), and tetracyclines (tetracycline: $30 \mu \mathrm{g}$ ). Among the all isolated enterococci a subset of pure $E$. faecalis and E. faecium culture $(n=117)$ representing individual positive samples were selected for antibiogram study. Isolates non-susceptible to three or more antibiotic classes were defined as MDR. Isolates non-susceptible to 7 or 8 of the antibiotics were termed as possible-Extremely Drug Resistant (XDR) isolates according to published criteria (Magiorakos et al. 2012). Isolates non-susceptible to vancomycin were termed vancomycin non-susceptible enterococci (VNSE).

\section{Statistics and data analysis}

Descriptive statistics and univariate analysis were performed using SPSS Statistics 20 software (IBM corp, USA) and The Survey System 12.0 (Creative Research Systems, USA). Inhibition zone data in antibiotic susceptibility assay were analyzed using BacLink and WHONET-2019 software (WHO Collaborating Centre for Surveillance of Antimicrobial Resistance, USA) (Stelling et al. 2007). Comparative analysis of antibiotic resistance was done using online data analysis tool 'Morpheus' created on R Studio interface to identify isolates that exhibited dual antibiotic resistance patterns (Morpheus 2018). For this analysis the cattle, goat, and camel samples were combined to form a group termed 'Livestock' as farming system for these species are largely similar in Bangladesh. Moreover, same butcher shop sells all these meat species sharing the same slaughtering facilities and equipment and similar processing techniques. A $p$ value of $\leq 0.05$ was considered significant.

\section{Antibiotic resistance gene and virulence factors identification}

The presence of the vancomycin resistance genes vanA, vanB, van $\mathrm{C}_{1}$ and van $_{2} / \mathrm{C}_{3}$ were determined using a multiplex PCR (m-PCR II) according to previously published protocol. Published PCR primers were also used to assess the presence of ten virulence factors: aggregation substance (asa1), gelatinase (ge/E), cytolysin (cy/A), enterococcal surface protein (esp), hyaluronidase (hyl), collagen-binding protein (ace), transmembrane protein ( $f s / \mathrm{B})$, endocarditis specific antigen (efa), aggregation protein (agg), and sex pheromones (cpd) (Strateva et al. 2016; Vankerckhoven et al. 2004; Zoletti et al. 2011). All PCR primers used are given in (Supplementary material 1 )

\section{Results}

Isolation of Enterococcus spp.

A total of 211 Enterococcus spp. comprising 115 (105 single and 10 co-isolation) E. faecalis, 36 (26 single and 10 co-isolation) E. faecium and 60 enterococci not assigned to a species by PCR were isolated from 352 collected samples, summarized in Table 1 and detailed in Supplementary material 2. Overall prevalence of Enterococcus spp. was $57 \%$ (95\%, Cl 52-62). In poultry, farm samples yielded significantly higher recovery of enterococci $(\mathrm{p}<0.05)$ compared to poultry meat samples of retail markets. Conversely, for livestock a greater proportion of meat samples were positive for enterococci compared to fecal samples (Table 1). In case of samples collected on farm, a significantly greater proportion of poultry isolates were culture positive for $E$. faecalis compared to livestock, however, retail livestock meat carried the greater proportion of E. faecalis than poultry meat (Table 1). 
Table 1

Prevalence of enterococci in corresponding farm and fresh meat samples. Significant $p$-value are marked as bc

\begin{tabular}{|c|c|c|c|c|c|c|c|c|c|c|c|c|c|c|c|c|}
\hline \multirow{3}{*}{$\begin{array}{l}\text { Isolates } \\
\\
\text { n (\%) }\end{array}$} & \multicolumn{7}{|c|}{ Poultry (n=196) } & \multicolumn{7}{|c|}{ Livestock ( $\mathrm{n=156)}$} & \multirow{2}{*}{$\begin{array}{l}\mathrm{p}- \\
\text { value }\end{array}$} & \multirow{3}{*}{$\begin{array}{l}\text { Ris! } \\
\text { Esti } \\
\text { OR }\end{array}$} \\
\hline & \multicolumn{2}{|c|}{ Farm $(n=136)$} & \multicolumn{2}{|c|}{ Meat $(n=60)$} & \multirow{2}{*}{$\begin{array}{l}\begin{array}{l}\text { p- } \\
\text { value }\end{array} \\
95 \% \\
\mathrm{Cl}\end{array}$} & \multicolumn{2}{|c|}{$\begin{array}{l}\text { Risk } \\
\text { Estimate }\end{array}$} & \multicolumn{2}{|c|}{ Farm $(n=94)$} & \multicolumn{2}{|c|}{ Meat $(n=62)$} & \multirow[t]{2}{*}{$\begin{array}{l}\mathrm{p}- \\
\text { value }\end{array}$} & \multicolumn{2}{|c|}{$\begin{array}{l}\text { Risk } \\
\text { Estimate }\end{array}$} & & \\
\hline & $95 \% \mathrm{Cl}$ & $\mathrm{n}(\%)$ & $95 \% \mathrm{Cl}$ & OR & & $\begin{array}{l}n \\
(\%)\end{array}$ & $\begin{array}{l}95 \% \\
\mathrm{Cl}\end{array}$ & $\begin{array}{l}n \\
(\%)\end{array}$ & $\begin{array}{l}95 \% \\
\mathrm{Cl}\end{array}$ & OR & $\begin{array}{l}95 \% \\
\mathrm{Cl}\end{array}$ & & & OR & $\begin{array}{l}95 \% \\
\mathrm{Cl}\end{array}$ & \\
\hline E. faecalis & $\begin{array}{l}50 \\
(36.8 \%)\end{array}$ & $\begin{array}{l}28.7- \\
44.9 \%\end{array}$ & $\begin{array}{l}12 \\
(20.0 \%)\end{array}$ & $\begin{array}{l}10.0- \\
31.6 \%\end{array}$ & .021 & 2.3 & $\begin{array}{l}1.1- \\
4.8\end{array}$ & $\begin{array}{l}18 \\
(19.1 \%)\end{array}$ & $\begin{array}{l}11.7- \\
27.7 \%\end{array}$ & $\begin{array}{l}25 \\
(40.3 \%)\end{array}$ & $\begin{array}{l}29.0- \\
51.6 \%\end{array}$ & .006 & 0.4 & $\begin{array}{l}0.2- \\
0.7\end{array}$ & .415 & 1.2 \\
\hline E. faecium & $\begin{array}{l}10 \\
(7.4 \%)\end{array}$ & $\begin{array}{l}3.7- \\
12.5 \%\end{array}$ & $\begin{array}{l}6 \\
(10.0 \%)\end{array}$ & $\begin{array}{l}3.3- \\
18.3 \%\end{array}$ & .575 & 0.7 & $\begin{array}{l}0.2- \\
2.1\end{array}$ & $\begin{array}{l}08 \\
(8.5 \%)\end{array}$ & $\begin{array}{l}3.2- \\
14.9 \%\end{array}$ & $\begin{array}{l}2 \\
(3.2 \%)\end{array}$ & $\begin{array}{l}0- \\
8.1 \%\end{array}$ & .317 & 2.7 & $\begin{array}{l}0.6- \\
13.6\end{array}$ & .682 & 1.3 \\
\hline $\begin{array}{l}\text { E. faecalis } \\
\& \text { E. } \\
\text { faecium) }\end{array}$ & $\begin{array}{l}5 \\
(3.7 \%)\end{array}$ & $\begin{array}{l}0.7- \\
7.4 \%\end{array}$ & $\begin{array}{l}0 \\
(0.0 \%)\end{array}$ & $0-0 \%$ & .326 & - & - & $\begin{array}{l}4 \\
(4.3 \%)\end{array}$ & $\begin{array}{l}1.1- \\
8.5 \%\end{array}$ & $\begin{array}{l}1 \\
(1.6 \%)\end{array}$ & $\begin{array}{l}0- \\
4.8 \%\end{array}$ & .649 & 2.7 & $\begin{array}{l}0.3- \\
24.8\end{array}$ & .755 & 0.8 \\
\hline $\begin{array}{l}\text { Other } \\
\text { Enterococci }\end{array}$ & $\begin{array}{l}19 \\
(14.0 \%)\end{array}$ & $\begin{array}{l}9.7- \\
19.5 \%\end{array}$ & $\begin{array}{l}3 \\
(5.0 \%)\end{array}$ & $\begin{array}{l}1.1- \\
11.3 \%\end{array}$ & .055 & 1.9 & $\begin{array}{l}0.8- \\
3.7\end{array}$ & $\begin{array}{l}28 \\
(29.8 \%)\end{array}$ & $\begin{array}{l}23.7- \\
36.6 \%\end{array}$ & $\begin{array}{l}10 \\
(16.1 \%)\end{array}$ & $\begin{array}{l}29.0- \\
51.6 \%\end{array}$ & .125 & 1.8 & $\begin{array}{l}0.7- \\
3.9\end{array}$ & .335 & 1.3 \\
\hline Overall & $\begin{array}{l}84 \\
(61.8 \%)\end{array}$ & $\begin{array}{l}53.7- \\
68.5 \%\end{array}$ & $\begin{array}{l}21 \\
(35.0 \%)\end{array}$ & $\begin{array}{l}18.9- \\
41 \%\end{array}$ & .028 & 2.1 & $\begin{array}{l}1.1- \\
4.1\end{array}$ & $\begin{array}{l}58 \\
(61.7 \%)\end{array}$ & $\begin{array}{l}52.8- \\
69.3 \%\end{array}$ & $\begin{array}{l}38 \\
(61.3 \%)\end{array}$ & $\begin{array}{l}52.0- \\
70.1 \%\end{array}$ & .087 & 0.5 & $\begin{array}{l}0.2- \\
1.1\end{array}$ & .381 & 1.1 \\
\hline
\end{tabular}

\section{Antimicrobial Susceptibility}

High prevalence of VNSE (44\%) was observed in both farm (41\%) and meat (52\%) isolates, in addition, resistance level was also noteworthy for tetracycline (74\%) and erythromycin (65\%). Moreover, twenty isolates (17\%) were resistant to linezolid which includes 14 and 6 isolates from farm and meat respectively. MDR was observed in $80 \%$ of the isolates with a similar proportion for both E. faecalis (79\%) and E. faecium (81\%). One E. faecium isolate and seven E. faecalis isolates were possible-XDR, having resistance to $\geq 7$ antibiotic classes. Almost all the $E$. faecalis and $E$. faecium from poultry, including poultry farms $(n=57 / 60)$ and poultry meat $(n=9 / 9)$ were MDR. The full antibiotic resistance patterns and profiles towards the eight antimicrobials tested is presented in Table 2 and Figure 1 
Table 2

Antibiotic resistance of enterococci among tested poultry, poultry meat, livestock and livestock meat isolates

\begin{tabular}{|c|c|c|c|c|c|c|c|c|c|c|}
\hline \multirow{2}{*}{$\begin{array}{l}\text { Enterococci } \\
\text { Sources }\end{array}$} & \multirow[t]{2}{*}{ Isolates } & \multicolumn{9}{|c|}{ Antimicrobials } \\
\hline & & $\mathrm{CHL}$ & CIP & ERY & NIT & LID & PEN & TET & VAN & MDR \\
\hline \multirow[t]{6}{*}{ Poultry } & E. faecalis $(\mathrm{n}=46)$ & 9 & 17 & 38 & 6 & 8 & 21 & 44 & $9(20 \%)$ & 43 (93\%) \\
\hline & & $(20 \%)$ & $(37 \%)$ & $(83 \%)$ & $(13 \%)$ & $(17 \%)$ & $(46 \%)$ & $(96 \%)$ & & \\
\hline & E. faecium $(\mathrm{n}=14)$ & 1 & 6 & $11(79 \%)$ & 2 & 0 & 3 & 13 & 1 & $14(100 \%)$ \\
\hline & & $(7 \%)$ & $(43 \%)$ & & $(14 \%)$ & $(0 \%)$ & $(21 \%)$ & $(93 \%)$ & $(7 \%)$ & \\
\hline & Overall $(n=60)$ & 10 & 23 & 49 & 8 & 8 & 24 & 57 & 10 & 57 (95\%) \\
\hline & & $(17 \%)$ & $(38 \%)$ & $(82 \%)$ & $(13 \%)$ & $(13 \%)$ & $(40 \%)$ & $(95 \%)$ & $(17 \%)$ & \\
\hline \multirow[t]{6}{*}{ Poultry Meat } & E. faecalis $(\mathrm{n}=5)$ & 2 & 2 & 5 & 2 & 3 & 2 & 4 & 3 & $5(100 \%)$ \\
\hline & & $(40 \%)$ & $(40 \%)$ & $(100 \%)$ & $(40 \%)$ & $(60 \%)$ & $(40 \%)$ & $(80 \%)$ & $(60 \%)$ & \\
\hline & E. faecium $(\mathrm{n}=4)$ & 1 & 2 & 4 & 1 & 1 & 2 & 4 & 1 & $4(100 \%)$ \\
\hline & & $(25 \%)$ & $(50 \%)$ & $(100 \%)$ & $(25 \%)$ & $(25 \%)$ & $(50 \%)$ & $(100 \%)$ & $(25 \%)$ & \\
\hline & Overall $(n=9)$ & $3(33 \%)$ & $4(44 \%)$ & 9 & 3 & 4 & 4 & 8 & $4(44 \%)$ & $9(100 \%)$ \\
\hline & & & & $(100 \%)$ & $(33 \%)$ & $(44 \%)$ & $(44 \%)$ & $(89 \%)$ & & \\
\hline \multirow[t]{6}{*}{ Livestock } & E. faecalis $(n=15)$ & 0 & 2 & 7 & 0 & 3 & 0 & 7 & 2 & $9(60 \%)$ \\
\hline & & $(0 \%)$ & $(13 \%)$ & $(47 \%)$ & $(0 \%)$ & $(20 \%)$ & $(0 \%)$ & $(47 \%)$ & $(13 \%)$ & \\
\hline & E. faecium $(n=11)$ & 0 & 0 & 6 & 0 & 3 & 1 & $5(45 \%)$ & $2(18 \%)$ & $6(55 \%)$ \\
\hline & & $(0 \%)$ & $(0 \%)$ & $(55 \%)$ & $(0 \%)$ & $(27 \%)$ & $(9 \%)$ & & & \\
\hline & Overall $(n=26)$ & 0 & 2 & 13 & 0 & 6 & 1 & 12 & $4(15 \%)$ & $15(58 \%)$ \\
\hline & & $(0 \%)$ & $(8 \%)$ & $(50 \%)$ & $(0 \%)$ & $(23 \%)$ & $(4 \%)$ & $(46 \%)$ & & \\
\hline \multirow[t]{6}{*}{ Livestock's Meat } & E. faecalis $(n=20)$ & 0 & 7 & 4 & 1 & 2 & 2 & 9 & 0 & $11(55 \%)$ \\
\hline & & $(0 \%)$ & $(35 \%)$ & $(20 \%)$ & $(5 \%)$ & $(10 \%)$ & $(10 \%)$ & $(45 \%)$ & $(0 \%)$ & \\
\hline & E. faecium $(\mathrm{n}=2)$ & 0 & 1 & 1 & 0 & 0 & 0 & 1 & 0 & $1(50 \%)$ \\
\hline & & $(0 \%)$ & $(50 \%)$ & $(50 \%)$ & $(0 \%)$ & $(0 \%)$ & $(0 \%)$ & $(50 \%)$ & $(0 \%)$ & \\
\hline & Overall $(n=22)$ & 0 & 8 & 5 & 1 & 2 & 2 & 10 & 0 & $12(55 \%)$ \\
\hline & & $(0 \%)$ & $(36 \%)$ & $(23 \%)$ & $(5 \%)$ & $(9 \%)$ & $(9 \%)$ & $(46 \%)$ & $(0 \%)$ & \\
\hline \multirow[t]{4}{*}{ Overall } & E. faecalis $(\mathrm{n}=86)$ & 11 & 28 & 54 & 9 & 16 & 25 & 64 & 14 & 68 \\
\hline & & $(13 \%)$ & $(33 \%)$ & $(63 \%)$ & $(10 \%)$ & $(19 \%)$ & $(29 \%)$ & $(74 \%)$ & $(16 \%)$ & $(79 \%)$ \\
\hline & E. faecium $(\mathrm{n}=31)$ & 2 & 9 & 22 & 3 & 4 & 6 & 23 & 4 & 25 \\
\hline & & $(6 \%)$ & $(29 \%)$ & $(71 \%)$ & $(10 \%)$ & $(13 \%)$ & $(19 \%)$ & $(74 \%)$ & $(13 \%)$ & $(81 \%)$ \\
\hline \multirow{2}{*}{\multicolumn{2}{|c|}{ Overall Resistance $(n=117)$}} & 13 & 37 & 76 & 12 & 20 & 31 & 87 & 18 & 93 \\
\hline & & $(11 \%)$ & $(32 \%)$ & $(65 \%)$ & $(10 \%)$ & $(17 \%)$ & $(27 \%)$ & $(74 \%)$ & $(15 \%)$ & $(80 \%)$ \\
\hline
\end{tabular}

\section{Detection Of Antibiotic Resistance Genes}

A total of 51 VNSE isolates were further characterized to determine the presence of vancomycin resistance genes (Table 3). Twenty isolates were PCR positive for at least one vancomycin resistance gene, of which nine isolates were phenotypically resistance to vancomycin. Nonetheless, eleven isolates harbored at least one vancomycin resistance gene without being phenotypically resistant, though these were vancomycin non sensitive. The most commonly detected vancomycin resistance genes were vanA and van $_{2 / 3}$. 
Table 3

Resistance profile, vancomycin resistance gene and virulence factors observed in multidrug resistant isolates from different sources

\begin{tabular}{|c|c|c|c|c|c|c|c|}
\hline Sample ID & Source & Isolate & Resistance Profile & N. Res & VAN & Van resistance gene & Virulence gene \\
\hline BCS 016 & Pountry Cloaca & E. faecalis & ERY VAN & 2 & $\mathrm{R}$ & & $g e / \mathrm{E}, c p d$ \\
\hline LBM 002 & Beef & E. faecalis & ERY VAN & 2 & I & & asa1 \\
\hline LBM 011 & Beef & E. faecalis & ERY VAN & 2 & I & & asa 1, ge/E, cpd \\
\hline L-En 017 & Camel Fecal & E. faecium & ERY VAN & 2 & 1 & & \\
\hline LBM 014 & Beef & E. faecalis & CIP ERY VAN & 3 & 1 & & asa1, ge/E, cpd \\
\hline LBM 023 & Beef & E. faecalis & CIP ERY VAN & 3 & 1 & & asa1, ge/E \\
\hline LBM 028 & Beef & E. faecalis & CIP ERY VAN & 3 & I & & asa1, cpd \\
\hline L-En 028 & Camel Fecal & E. faecium & ERY TET VAN & 3 & I & & \\
\hline BBS 009 & Pountry Cloaca & E. faecalis & CIP ERY TET VAN & 4 & I & & $g e / \mathbb{E}, c p d$ \\
\hline BBS 012 & Pountry Cloaca & E. faecalis & CIP ERY TET VAN & 4 & I & & $c p d$ \\
\hline BCS 031 & Pountry Cloaca & E. faecalis & CIP ERY TET VAN & 4 & 1 & $\operatorname{van} \mathrm{C} 1, \operatorname{van} \mathrm{C} 2 / 3$ & $a s a 1, g e / \mathbb{E}, c p d$ \\
\hline BCS 032 & Pountry Cloaca & E. faecalis & CIP ERY TET VAN & 4 & I & $\operatorname{van} \mathrm{C} 1, \operatorname{van} \mathrm{C} 2 / 3$ & $g e / \mathbb{E}, c p d$ \\
\hline BCS 033 & Pountry Cloaca & E. faecalis & CIP ERY TET VAN & 4 & 1 & & $g e / E$ \\
\hline LBM 001 & Beef & E. faecalis & CIP ERY TET VAN & 4 & I & & $g e / \mathrm{E}, c p d$ \\
\hline LBM 016 & Beef & E. faecalis & CIP ERY LNZ VAN & 4 & I & & asa1, ge/E, cpd \\
\hline LCM 047 & Chicken Meat & E. faecalis & CIP ERY TET VAN & 4 & I & & $g e / \mathbb{E}, c p d$ \\
\hline LCS 006 & Pountry Cloaca & E. faecalis & CIP ERY TET VAN & 4 & 1 & & ge/E \\
\hline LCS 008 & Pountry Cloaca & E. faecalis & CIP ERY TET VAN & 4 & I & $\operatorname{vanA}$ & asa1, ge/E \\
\hline LCS 008 & Pountry Cloaca & E. faecium & CIP ERY TET VAN & 4 & I & vanA & asa1, ge/E \\
\hline LCS 011 & Pountry Cloaca & E. faecalis & CIP ERY TET VAN & 4 & I & & $g e / \mathrm{E}, c p d$ \\
\hline LCS 013 & Pountry Cloaca & E. faecalis & CIP ERY TET VAN & 4 & I & & asa1, ge/E, cpd \\
\hline LCS 032 & Pountry Cloaca & E. faecium & CIP ERY NIT VAN & 4 & $\mathrm{R}$ & & asa1, ge/E, cpd \\
\hline LCS 048 & Pountry Cloaca & E. faecalis & CIP ERY TET VAN & 4 & I & & \\
\hline L-En 009 & Camel Fecal & E. faecalis & CIP ERY LNZ VAN & 4 & I & $\operatorname{van} \mathrm{C} 2 / 3$ & $g e / \mathbb{E}, c p d$ \\
\hline L-En 014 & Camel Fecal & E. faecalis & ERY TET LNZ VAN & 4 & $\mathrm{R}$ & vanA & asa1, ge/E \\
\hline L-En 015 & Camel Fecal & E. faecium & ERY TET LNZ VAN & 4 & $\mathrm{R}$ & $\operatorname{vanA}$ & \\
\hline L-En 033 & Goat Fecal & E. faecalis & ERY TET LNZ VAN & 4 & I & $\operatorname{vanC1}$ & asa1, ge/E \\
\hline L-En 033 & Goat Fecal & E. faecium & ERY TET LNZ VAN & 4 & I & vanC1 & asa1, ge/E \\
\hline LMM 015 & Mutton & E. faecalis & CIP ERY TET VAN & 4 & 1 & & \\
\hline BBS 007 & Pountry Cloaca & E. faecalis & CHL CIP ERY TET VAN & 5 & 1 & & $g e / \mathbb{E}, c p d$ \\
\hline BCS 013 & Pountry Cloaca & E. faecalis & CHL CIP ERY TET VAN & 5 & 1 & & $g e / \mathrm{E}, c p d$ \\
\hline LCM 037 & Chicken Meat & E. faecium & CHL CIP ERY TET VAN & 5 & 1 & & \\
\hline L-En 027 & Camel Fecal & E. faecium & ERY PEN TET LNZ VAN & 5 & $\mathrm{R}$ & $\operatorname{van} \mathrm{B}$ & asa1, ge/E, cpd \\
\hline BCS 029 & Pountry Cloaca & E. faecalis & CHL ERY PEN TET LNZ VAN & 6 & $\mathrm{R}$ & & asa1, ge/E, cpd \\
\hline BCS 035 & Pountry Cloaca & E. faecalis & CHL CIP ERY NIT TET VAN & 6 & 1 & $\operatorname{vanC1}$ & asa1, ge/E \\
\hline BCS 035 & Pountry Cloaca & E. faecium & CHL CIP ERY NIT TET VAN & 6 & 1 & $\operatorname{vanC1}$ & asa1, ge/E \\
\hline LBM 022 & Beef & E. faecalis & CHL CIP ERY PEN TET VAN & 6 & I & & asa1, ge/E \\
\hline LCM 054 & Chicken Meat & E. faecalis & CIP ERY PEN TET LNZ VAN & 6 & $\mathrm{R}$ & & $g e / E, c p d$ \\
\hline LCM 057 & Chicken Meat & E. faecalis & CIP ERY NIT TET LNZ VAN & 6 & $\mathrm{R}$ & & $g e / \mathbb{E}, c p d$ \\
\hline
\end{tabular}

Note: N. Res: Number of antibiotic classes to which the corresponding isolate was non-susceptible. CHL: Chloramphenicol, CIP: Ciprofloxacin, ERY: Erythromycin, NIT: Nitrofurantoin, PEN: Penicillin G, TET: Tetracycline, LID: Linezolid, VAN: Vancomycin. R: Resistant, I: Intermediate. 


\begin{tabular}{|c|c|c|c|c|c|c|c|}
\hline Sample ID & Source & Isolate & Resistance Profile & N. Res & VAN & Van resistance gene & Virulence gene \\
\hline LCS 003 & Pountry Cloaca & E. faecalis & CIP ERY PEN TET LNZ VAN & 6 & $\mathrm{R}$ & & $g e / \mathrm{E}, c p d$ \\
\hline LCS 037 & Pountry Cloaca & E. faecalis & CIP ERY NIT TET LNZ VAN & 6 & $\mathrm{R}$ & & $g e / \mathrm{E}, c p d$ \\
\hline LCS 039 & Pountry Cloaca & E. faecalis & ERY NIT PEN TET LNZ VAN & 6 & $\mathrm{R}$ & & $g e / \mathbb{E}, c p d$ \\
\hline LCS 040 & Pountry Cloaca & E. faecalis & CIP ERY NIT TET LNZ VAN & 6 & $\mathrm{R}$ & $\operatorname{vanC} 2 / 3$ & $g e / \mathrm{E}, c p d$ \\
\hline BCS 030 & Pountry Cloaca & E. faecalis & CHL CIP ERY NIT PEN TET VAN & 7 & I & $\operatorname{vanC1}$ & $g e / \mathrm{E}$ \\
\hline LCM 040 & Chicken Meat & E. faecium & CIP ERY NIT PEN TET LNZ VAN & 7 & $\mathrm{R}$ & & $g e / E$ \\
\hline L-En 003 & Camel Fecal & E. faecalis & CHL CIP ERY NIT TET LNZ VAN & 7 & $\mathrm{R}$ & $\operatorname{van} \mathrm{A}, \operatorname{van} \mathrm{C} 2 / 3$ & $g e / \mathrm{E}, c p d$ \\
\hline LBM 025 & Beef & E. faecalis & CHL CIP ERY NIT PEN TET LNZ VAN & 8 & I & $\operatorname{van} \mathrm{A}$ & asa1, ge/E \\
\hline LCM 036 & Chicken Meat & E. faecalis & CHL CIP ERY NIT PEN TET LNZ VAN & 8 & $\mathrm{R}$ & $\operatorname{van} \mathrm{A}$ & $g e / \mathrm{E}, c p d$ \\
\hline LCS 016 & Pountry Cloaca & E. faecalis & CHL CIP ERY NIT PEN TET LNZ VAN & 8 & $\mathrm{R}$ & $\operatorname{vanC} 2 / 3$ & $g e / \mathrm{E}, c p d$ \\
\hline LCS 018 & Pountry Cloaca & E. faecalis & CHL CIP ERY NIT PEN TET LNZ VAN & 8 & $\mathrm{R}$ & vanc2/3 & asa1, ge/E, cpd \\
\hline LCS 029 & Pountry Cloaca & E. faecalis & CHL CIP ERY NIT PEN TET LNZ VAN & 8 & $\mathrm{R}$ & $\operatorname{van} \mathrm{A}, \operatorname{vanC} 2 / 3$ & cpd \\
\hline
\end{tabular}

Note: N. Res: Number of antibiotic classes to which the corresponding isolate was non-susceptible. CHL: Chloramphenicol, CIP: Ciprofloxacin, ERY: Erythromycin, NIT: Nitrofurantoin, PEN: Penicillin G, TET: Tetracycline, LID: Linezolid, VAN: Vancomycin. R: Resistant, I: Intermediate.

\section{Detection Of Virulence Genes}

The 51 VNSE isolates were also tested for the presence of ten virulence factors. The gelatinase gene (ge/E) was observed in 79\% isolates (42), aggregation factor (asa1) in $38 \%$ isolates (20), and the sex pheromones ( $c p d$ ) in $66 \%$ isolates (35) (Table 3 ). Other virulence factors, including cytolysin, surface protein, hyaluronidase, collagen-binding protein, transmembrane protein, endocarditis specific antigen, and aggregation protein were not observed among these isolates.

\section{Discussion}

To assess the prevalence of AMR enterococci in healthy finisher livestock and poultry, and their products in Bangladesh, we screened 230 samples from livestock and poultry at farms and 122 meat samples at retail markets. Our findings indicate that Enterococcus spp. are relatively common in poultry and livestock animals in Bangladesh, though the overall prevalence of E. faecium was lower than E. faecalis. Nonetheless, poultry has been demonstrated as the leading source of Enterococcus spp. in several studies conducted elsewhere (Maasjost et al. 2015; St邓pień-Pyśniak et al. 2016). The prevalence of E. faecalis was significantly higher in poultry farms than at other livestock farms, but retail livestock meat yielded a higher prevalence of $E$. faecalis than poultry meat. This is likely to be indicative of post-slaughter contamination as well as the absence of, or lack of enforcement of, food safety regulations during the slaughtering process and meat product handling in Bangladesh.

Poultry and livestock are essential to food security in Bangladesh, the potential dissemination of resistant Enterococcus spp. in the food production continuum and strengthening of food safety regulations needs to be addressed. In the context of One Health and to further inform food safety interventions in Bangladesh, it is also important to identify the major reservoirs and their dissemination downstream of the production continuum, which was the intent of our study. Our data indicates that more than half of the samples were contaminated with Enterococcus spp. and the most predominant species was $E$. faecalis, the third most commonly identified pathogen in hospitals associated with increased mortality (Coque 2008). These findings are consistent with several preceding studies (Ngbede et al. 2017; Poeta et al. 2006; Yoshimura et al. 2000). There is no extensive data available on AMR amongst enterococci in livestock and poultry in Bangladesh because a national AMR surveillance program has not yet been established. However, one study showed high prevalence of enterococci among chickens (Banik et al. 2018) consistent with our study. Two relevant studies in involving human cases have implicated enterococci (Akram Hossain 2016; Suchi et al. 2018).

High prevalence of VNSE $(44 \% ; n=51)$ has been observed in this study which is higher than some other studies (Cosentino et al. 2010; Maasjost et al. 2015; St $₫$ pień-Pyśniak et al. 2016). Nonetheless, most alarming finding was the co-occurrence of linezolid resistance and VNSE, where most linezolid (90\%) resistant isolates were VNSE, which could drive a therapeutic crisis for treating infection caused by VNSE (Bialvaei et al. 2017). In addition, this study detected low susceptibility of isolates to antimicrobials that are concurrently being used for both human infection and animal production in Bangladesh. In our study, the frequency of resistance to tetracyclines and macrolides were relatively high whereas comparatively low resistance were observed to DNA synthesis inhibitors including nitrofurantoin (10\%) and ciprofloxacin (31\%), which are consistent with similar studies conducted elsewhere (Cosentino et al. 2010; Maasjost et al. 2015; Stðpień-Pyśniak et al. 2016).

MDR prevalence was high in farm and in fresh meat sold at retail markets (i.e. products originating from the same geographical locations where they were raised) and most of the VNSE isolates were also MDR $(92 \%, 47 / 51)$. Remarkably, isolates that were possible-XDR were mostly recovered from poultry farms, this also raises the potential public health concern of exposure to and consumption of products from this species. These findings may be reflective of the 
indiscriminate use of antimicrobials in poultry in Bangladesh, (Ahmed et al. 2019) notably, the high use of CIAs (Imam et al. 2020) and thus support the need for surveillance of AMR and monitoring of AMU to inform changes in usage policy and to better understand AMR and AMU relationships. The comparative pairwise antibiotic resistance matrix reveals that the combination between chloramphenicol or ciprofloxacin with nitrofuratoin, linezolid or vancomycin may produce better efficiency against multidrug resistant isolates as combined resistance level to those antibiotics is very low compared to the other antibiotics (Figure 2). These findings warrant further research.

The presence of vanA gene in our study, primarily responsible for vancomycin resistance (Torres et al. 2018) further highlight the widespread dissemination of these resistant strains in animal populations. However, there was a discrepancy in detection of vancomycin resistance gene in our study; it was detected in both resistance and intermediate phenotypes, which might be showing that resistance is not dependent on gene presence alone rather depends on the gene expression level. We also found the presence of vanC1 and vanC2/3 gene among $E$. faecalis which was previously thought to be species specific to $E$. gallinarum and E. casseliflavus, respectively (Clark et al. 1998). The vanC gene cluster can be located on plasmids (as well as the chromosome), and can be transferred to other enterococci such as E. faecium and E. faecalis, (Moura et al. 2013; Sun et al. 2014) however the location of the vanC gene in the isolates from this study was not determined and may warrant further study in the future. We observed that most genotypic vancomycin-resistant enterococci (VRE) isolates were phenotypically non-resistant to vancomycin indicating vancomycin-variable enterococci (VVE), as described previously (Downing et al. 2015; Thaker et al. 2015). This could be a result of the mutation in van gene cassette including vanSR or vanHAXY gene cluster or any other novel mutations.(Hong et al. 2008) This particular finding has impact in clinical settings, where the misidentification in enterococcal infections may result to challenges in the development of an efficacious treatment regimen. The preceding studies found the relevance of VVE with only vanA gene, but in this study, we also found the association of vanB, vanC1 and vanC2/3 with VVE which would require further investigation. We also found some vancomycin resistant isolates without having any major VRE gene which may be due to the other type of resistance mechanism and beyond the scope of our current study. However, this discrepancy in vancomycin resistance pattern may evolves as a new therapeutic challenge for clinical setting. An immediate improvisation in diagnostic technique for VVE and continuous surveillance for VVE is utmost necessary as some other studies suggested the same (Downing et al. 2015; Szakacs et al. 2014).

Moreover, these VRE gene have been associated with vancomycin resistance and can easily be transferred to another susceptible isolates since there is a probability to be clustered in a mobile genetic elements like plasmid or transposons (Torres et al. 2018) and investigation of this in the future would be informative. This transfer could occur within livestock farms and their environment or throughout the food production chain from farm to fork as evidenced by the detection of MDR E. faecalis and E. faecium from diverse food animal species such as poultry, cattle, and goat and meat products/meat-derived products (Hammerum et al. 2010; Hoelzer et al. 2017).

The high prevalence of virulence indicators like gelatinase, sex pheromones, and aggregation factors were found among the MDR isolates. The presence of these factors in MDR enterococci can contribute to the colonization or formation of bacterial biofilm-like vegetations among immuno-compromised patients through urinary tract or blood and subsequently turned into untreatable urinary tract infection (UTI) or endocarditis respectively (Sharifi et al. 2013).

\section{Conclusion}

Our study indicated that the livestock and poultry fresh meat are frequently contaminated with Enterococcus spp., possibly due to insufficient food safety practices in the slaughtering and meat processing systems in Bangladesh. Mostly poultry meat samples were contaminated with $E$. faecalis indicative that poultry is potentially an important source of resistant enterococci that can infect people via the food chain. The presence of enterococci resistant to WHO's CIA in food animals can pose an unprecedented threat to public health. The most alarming finding was the co-occurrence of vancomycin and linezolid resistance in enterococci in livestock and poultry. Moreover, the prevalence of gelatinase, pheromones and aggregation factor in MDR enterococci, in the face of poorly enforced (or lack of) food safety regulations in livestock and poultry in Bangladesh could plausibly lead to their widespread dissemination and persistence in nature. This study calls for an urgent need to reduce the use of WHO's CIA in livestock and poultry and enhancement of food safety practices at the farms and slaughter plants in Bangladesh to reduce the public health implications of Enterococcus spp., particularly VRE.

\section{Declarations}

\section{Acknowledgements}

The authors would like to express their thanks to the participating investigators and laboratories and the staff at ARAC for their work and the Phoenix poultry farm for technical support.

\section{Authors' contributions}

Conceptualization: Mohammed A. Samad; Methodology, Formal Analysis and investigation: Md Shahjalal Sagor, Muhammad Sazzad Hossain, Mohammad Asheak Mahmud, Md Samun Sarker; Writing - original draft preparation: Mohammed A. Samad, Md Shahjalal Sagor; Writing - review and editing: Fahria A. Shownaw, Md Rezaul Karim, Zakaria Mia, Md Samun Sarker, Roderick M. Card, Agnes Agunos, Lindahl Johanna; Funding acquisition: Mohammed A. Samad; Resources: Mohammed A. Samad; Supervision: Mohammed A. Samad, Lindahl Johanna.

\section{Funding}

The study was conducted with financial assistance of Ministry of Fisheries and Livestock, Bangladesh and US CDC (Grant number NU2GGH002077).

Code or data availability: On Request 
Ethical Approval: This study was conducted under the ethical regulation constructed by Bangladesh Livestock Research Institute (ARAC: 01/10/2016:01).

Consent to participate: Verbal consent from the authority of each farm was taken before collecting the samples from the farm.

Consent for publication: We give our consent for the publication of the submitted manuscript.

\section{References}

1. Santestevan NA, de Angelis Zvoboda D, Prichula J, Pereira RI, Wachholz GR, Cardoso LA et al (2015) Antimicrobial resistance and virulence factor gene profiles of Enterococcus spp. isolates from wild Arctocephalus australis (South American fur seal) and Arctocephalus tropicalis (Subantarctic fur seal). World J Microbiol Biotechnol 31(12):1935-1946. https://doi.org/10.1007/s11274-015-1938-7

2. Wang Y, Lv Y, Cai J, Schwarz S, Cui L, Hu Z et al (2015) A novel gene, optrA, that confers transferable resistance to oxazolidinones and phenicols and its presence in Enterococcus faecalis and Enterococcus faecium of human and animal origin. J Antimicrob Chemother 70(8):2182-2190. https://doi.org/10.1093/jac/dkv116

3. Arias CA, Murray BE (2012) The rise of the Enterococcus: Beyond vancomycin resistance. Nat Rev Microbiol 10(4):266-278. https://doi.org/10.1038/nrmicro2761

4. Lebreton F, Willems RJL, Gilmore MS (2014) Enterococcus diversity, origins in nature, and gut colonization. Enterococci: from commensals to leading causes of drug resistant infection. Massachusetts Eye and Ear Infirmary, Boston, p 24649513

5. Hollenbeck BL, Rice LB (2012) Intrinsic and acquired resistance mechanisms in enterococcus. Virulence 3(5):421-569. https://doi.org/10.4161/viru.21282

6. Kolář M (2018) Vancomycin-resistant enterococci. Klin Mikrobiol Infekc Lek 24(2):50-56. PMID: 30747432

7. Cassenego APV, d'Azevedo PA, Ribeiro AML, Frazzon J, Van Der Sand ST, Frazzon APG (2011) Species distribution and antimicrobial susceptibility of enterococci isolated from broilers infected experimentally with Eimeria spp and fed with diets containing different supplements. Brazilian $\mathrm{J}$ Microbiol 42(2):480-8. https://doi.org/10.1590/s1517-838220110002000012

8. Bennani M, Amarouch H, Oubrim N, Cohen N (2012) Identification and antimicrobial resistance of fecal enterococci isolated in coastal mediterranean environments of Morocco. Eur J Sci Res 70(2):266-275

9. Torres C, Alonso CA, Ruiz-Ripa L, León-Sampedro R, Del Campo R, Coque TM (2018) Antimicrobial Resistance in Enterococcus spp. of animal origin. Microbiol Spectr 6(4). https://doi.org/10.1128/microbiolspec.arba-0032-2018

10. López-Salas P, Llaca-Díaz J, Morfin-Otero R, Tinoco JC, Rodriguez-Noriega E, Salcido-Gutierres L et al (2013) Virulence and antibiotic resistance of enterococcus faecalis clinical isolates recovered from three states of mexico. Detection of linezolid resistance. Arch Med Res 44(6):422-8. https://doi.org/10.1016/j.arcmed.2013.07.003

11. Aslam M, Diarra MS, Checkley S, Bohaychuk V, Masson L (2012) Characterization of antimicrobial resistance and virulence genes in Enterococcus spp. isolated from retail meats in Alberta, Canada. Int J Food Microbiol 156(3):222-230. http://dx.doi.org/10.1016/j.ijfoodmicro.2012.03.026

12. Mannu L, Paba A, Daga E, Comunian R, Zanetti S, Duprè l et al (2003) Comparison of the incidence of virulence determinants and antibiotic resistance between Enterococcus faecium strains of dairy, animal and clinical origin. Int J Food Microbiol 88(2-3):291-304. https://doi.org/10.1016/s01681605(03)00191-0

13. Vignaroli C, Zandri G, Aquilanti L, Pasquaroli S, Biavasco F (2011) Multidrug-resistant enterococci in animal meat and faeces and Co-transfer of resistance from an Enterococcus durans to a human Enterococcus faecium. Curr Microbiol 62(5):1438-1447. https://doi.org/10.1007/s00284-011-9880$x$

14. Tyson GH, Nyirabahizi E, Crarey E, Kabera C, Lam C, Rice-Trujillo C et al (2018) Prevalence and antimicrobial resistance of enterococci isolated from retail meats in the United States, 2002 to 2014. Appl Environ Microbiol 84(1):e01902-17. https://doi.org/10.1128/aem.01902-17

15. Boehm AB, Sassoubre LM (2014) Enterococci as Indicators of Environmental Fecal Contamination. Feb 5. In: Gilmore MS, Clewell DB, Ike Y, Shankar N (eds) Enterococci: From Commensals to Leading Causes of Drug Resistant Infection. Massachusetts Eye and Ear Infirmary, Boston, p 24649503

16. Ogier J-C, Serror P (2008) Safety assessment of dairy microorganisms: the Enterococcus genus. Int J Food Microbiol 126(3):291-301. https://doi.org/10.1016/j.ijfoodmicro.2007.08.017

17. Hammerum AM, Lester CH, Heuer OE (2010) Antimicrobial-resistant enterococci in animals and meat: A human health hazard? Foodborne Pathog Dis 7(10):1137-1146. https://doi.org/10.1089/fpd.2010.0552

18. Hoelzer K, Wong N, Thomas J, Talkington K, Jungman E, Coukell A (2017) Antimicrobial drug use in food-producing animals and associated human health risks: What, and how strong, is the evidence? BMC Vet Res 13(1):1-38. https://doi.org/10.1186/s12917-017-1131-3

19. DGDB (2018) Directorate General of Drug Administration Allopathic Drug Database. Bangladesh: http://www.dgda.gov.bd/

20. World Health Organisation (2019) WHO list of critically important antimicrobials for human medicine (WHO CIA list). World Heal Organ 5th revision

21. Kuiken T, Leighton FA, Fouchier RA, LeDuc JW, Peiris JS, Schudel A, Stöhr K, Osterhaus AD (2005) Public health. Pathogen surveillance in animals. Science 9(5741):1680-1. https://doi.org/10.1126/science.1113310

22. Guerrero-Ramos E, Molina-González D, Blanco-Morán S, Igrejas G, Poeta P, Alonso-Calleja C et al (2016) Prevalence, antimicrobial resistance, and genotypic characterization of vancomycin-resistant enterococci in meat preparations. J Food Prot 79(5):748-756. https://doi.org/10.4315/0362-028x.jfp$15-390$ 
23. Jackson CR, Fedorka-Cray PJ, Barrett JB (2004) Use of a genus- and species-specific multiplex PCR for identification of enterococci. J Clin Microbiol 42(8):3558-3565. https://doi.org/10.1128/jcm.42.8.3558-3565.2004

24. Poyart C, Quesnes G, Trieu-Cuot $P$ (2000) Sequencing the gene encoding manganese-dependent superoxide dismutase for rapid species identification of enterococci. J Clin Microbiol 38(1):415-8. https://doi.org/10.1128/jcm.38.1.415-418.2000

25. Huq A, Haley BJ, Taviani E, Chen A, Hasan NA, Colwell RR (2012) Detection, isolation, and identification of Vibrio cholerae from the environment. Curr Protoc Microbiol 6(6 A.5). https://doi.org/10.1002/9780471729259.mc06a05s26

26. Tuohy MJ, Procop GW, Washington JA (2000) Antimicrobial susceptibility of Abiotrophia adiacens and Abiotrophia defectiva. Diagn Microbiol Infect Dis 38(3):189-191. https://doi.org/10.1016/s0732-8893(00)00194-2

27. Magiorakos AP, Srinivasan A, Carey RB, Carmeli Y, Falagas ME, Giske CG et al (2012) Multidrug-resistant, extensively drug-resistant and pandrug-resistant bacteria: An international expert proposal for interim standard definitions for acquired resistance. Clin Microbiol Infect 18(3):268-281. http://dx.doi.org/10.1111/j.1469-0691.2011.03570.x

28. Stelling JM, Kulldorff M, O 'brien TF (2007) WHONET and BacLink: Software Tools for Laboratory-Based Surveillance of Infectious Diseases and Antimicrobial Resistance. Advances in Disease Surveillance 2:121

29. Morpheus (2018) https://software.broadinstitute.org/morpheus

30. Vankerckhoven V, Van Autgaerden T, Vael C, Lammens C, Chapelle S, Rossi R et al (2004) Development of a multiplex PCR for the detection of asal, gelE, cylA, esp, and hyl genes in enterococci and survey for virulence determinants among european hospital isolates of Enterococcus faecium. $J$ Clin Microbiol 42(10):4473-9. https://doi.org/10.1128/jcm.42.10.4473-4479.2004

31. Strateva T, Atanasova D, Savov E, Petrova G, Mitov I (2016) Incidence of virulence determinants in clinical Enterococcus faecalis and Enterococcus faecium isolates collected in Bulgaria. Brazilian J Infect Dis 20(2):127-133. http://dx.doi.org/10.1016/j.bjid.2015.11.011

32. Zoletti GO, Pereira EM, Schuenck RP, Teixeira LM, Siqueira JF, Dos Santos KRN (2011) Characterization of virulence factors and clonal diversity of Enterococcus faecalis isolates from treated dental root canals. Res Microbiol 162(2):151-8. https://doi.org/10.1016/j.resmic.2010.09.018

33. St『pień-Pyśniak D, Marek A, Banach T, Adaszek $Ł$, Pyzik E, Wilczyński J et al (2016) Prevalence and antibiotic resistance of enterococcus strains isolated from poultry. Acta Vet Hung 64(2):148-163. https://doi.org/10.1556/004.2016.016

34. Maasjost J, Mühldorfer K, De Jäckel SC, Hafez HM (2015) Antimicrobial susceptibility patterns of enterococcus faecalis and enterococcus faecium isolated from poultry flocks in Germany. Avian Dis 59(1):143-8. https://doi.org/10.1637/10928-090314-regr

35. Coque TM (2008) Evolutionary biology of pathogenic enterococci. In: Baquero F, Nombela C, Cassell GH, Guitierrez JA (eds) Evolutionary biology of bacterial and fungal pathogens. ASM Press, Washington, DC, pp 501-521

36. Yoshimura H, Ishimaru M, Endoh YS, Kojima A (2000) Antimicrobial susceptibilities of enterococci isolated from faeces of broiler and layer chickens. Lett Appl Microbiol 31(6):427-432. https://doi.org/10.1046/j.1365-2672.2000.00842.x

37. Poeta P, Costa D, Rodrigues J, Torres C (2006) Antimicrobial resistance and the mechanisms implicated in faecal enterococci from healthy humans, poultry and pets in Portugal. Int J Antimicrob Agents 27(2):131-7. https://doi.org/10.1016/j.jjantimicag.2005.09.018

38. Ngbede EO, Raji MA, Kwanashie CN, Kwaga JKP (2017) Antimicrobial resistance and virulence profile of enterococci isolated from poultry and cattle sources in Nigeria. Trop Anim Health Prod 49(3):451-8. http://dx.doi.org/10.1007/s11250-016-1212-5

39. Banik A, Mohammad N, Akter T, Fatema K, Abony M (2018) Prevalence, Identification and Antibiotic Susceptibility of Enterococcus Species Isolated from Chicken and Pigeon Meat in Gazipur Area of Bangladesh. Open J Med Microbiol 08(03):74-83. https://doi.org/10.4236/ojmm.2018.83007

40. Akram Hossain M (2016) Antimicrobial Susceptibility Pattern of Enterococci Isolated from Clinical Specimens at Mymensingh Medical College Hospital, Mymensingh, Bangladesh. J Bacteriol Mycol Open Access 3(3). https://doi.org/10.15406/jbmoa.2016.03.00061

41. Suchi SE, Shamsuzzaman S, Uddin BMM, Yusuf MA (2018) Detection of Virulence Factors and Antimicrobial Resistance in Enterococci Isolated from Urinary Tract Infection. Bangladesh J Infect Dis 4(2):30-34. http://dx.doi.org/10.3329/bjid.v4i2.37682

42. Cosentino S, Podda GS, Corda A, Fadda ME, Deplano M, Pisano MB (2010) Molecular detection of virulence factors and antibiotic resistance pattern in clinical Enterococcus faecalis strains in Sardinia. J Prev Med Hyg 51(1):31-36. PMID: 20853674.

43. Bialvaei AZ, Rahbar M, Yousefi M, Asgharzadeh M, Kafil HS (2017) Linezolid: A promising option in the treatment of Gram-positives. J Antimicrob Chemother 72(2):354-364. https://doi.org/10.1093/jac/dkw450

44. Ahmed I, Rabbi MB, Sultana S (2019) Antibiotic resistance in Bangladesh: A systematic review. Int J Infect Dis 80:54-61. https://doi.org/10.1016/j.jijid.2018.12.017

45. Imam T, Gibson JS, Foysal M, Das SB, Gupta S, Das, Fournié G et al (2020) A Cross-Sectional Study of Antimicrobial Usage on Commercial Broiler and Layer Chicken Farms in Bangladesh. Front Vet Sci 7(December):1-11. https://doi.org/10.3389/fvets.2020.576113

46. Clark NC, Teixeira LM, Facklam RR, Tenover FC (1998) Detection and differentiation of vanC-1, vanC-2, and vanC-3 glycopeptide resistance genes in enterococci. J Clin Microbiol 36(8):2294-7. https://doi.org/10.1128/jcm.36.8.2294-2297.1998

47. de Moura TM, Cassenego APV, Campos FS, Ribeiro AML, Franco AC, d'Azevedo PA et al (2013) Detection of vanC 1 gene transcription in vancomycinsusceptible Enterococcus faecalis. Mem Inst Oswaldo Cruz 108(4):453-6. https://doi.org/10.1590/s0074-0276108042013009

48. Sun M, Wang Y, Chen Z, Zhu X, Tian L, Sun Z (2014) The first report of the vanC1 gene in Enterococcus faecium isolated from a human clinical specimen. Mem Inst Oswaldo Cruz 109(6):712-5. https://doi.org/10.1590/0074-0276140019

49. Thaker MN, Kalan L, Waglechner N, Eshaghi A, Patel SN, Poutanen S et al (2015) Vancomycin-variable enterococci can give rise to constitutive resistance during antibiotic therapy. Antimicrob Agents Chemother 59(3):1405-1410. https://doi.org/10.1128/aac.04490-14 
50. Downing MA, Xiong J, Eshaghi A, McGeer A, Patel SN, Johnstone J (2015) Vancomycin-variable enterococcal bacteremia. J Clin Microbiol 53(12):3951-3. https://doi.org/10.1128/jcm.02046-15

51. Hong HJ, Hutchings MI, Buttner MJ (2008) Vancomycin resistance VanS/VanR two-component systems. Adv Exp Med Biol 631:200-213. https://doi.org/10.1007/978-0-387-78885-2_14

52. Szakacs TA, Kalan L, McConnell MJ, Eshaghi A, Shahinas D, McGeer A et al (2014) Outbreak of vancomycin-susceptible Enterococcus faecium containing the wild-type vanA gene. J Clin Microbiol 52(5):1682-6. https://doi.org/10.1128/jcm.03563-13

53. Sharifi Y, Hasani A, Ghotaslou R, Naghili B, Aghazadeh M, Milani M et al (2013) Virulence and antimicrobial resistance in enterococci isolated from urinary tract infections. Adv Pharm Bull 3(1):197-201. https://doi.org/10.5681/apb.2013.032

\section{Figures}

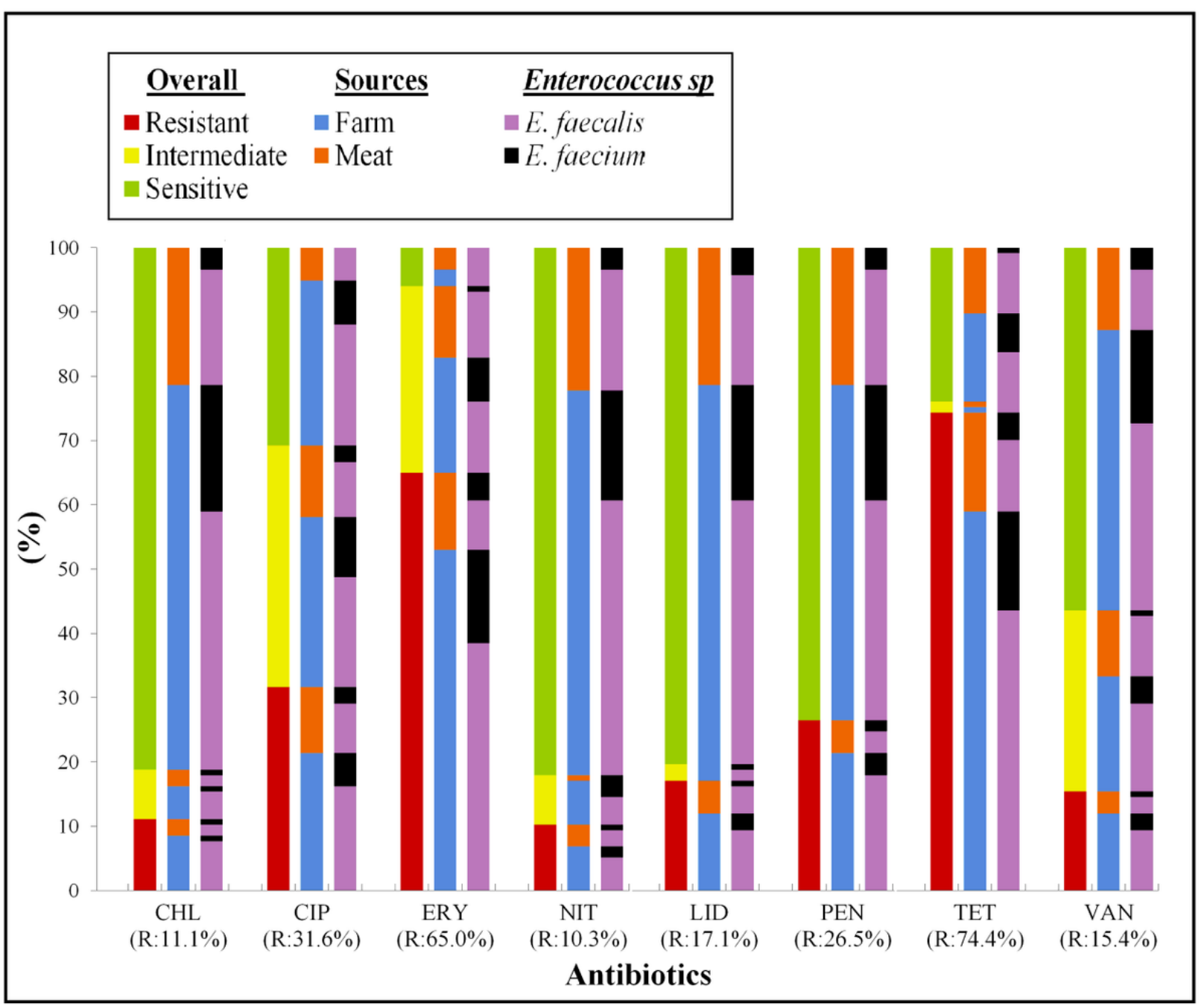

Figure 1

Overall antimicrobial resistance of Enterococci isolates against each antibiotic: 117 isolates comprising 86 E. faecalis and $31 \mathrm{E}$. faecium originated from farm $(n=86)$ and meat $(n=31)$, were tested against 8 antibiotics. Of three vertically related adjacent bars for each antibiotic, the left bar representing the overall antimicrobial sensitivity of corresponding antibiotic against all isolates; middle bar shows the origin of resistant, intermediate or sensitive isolates; and the right bar express the species of isolates (E. faecalis or E. faecium) themselves related to the source and antimicrobial criteria. Note: R: Resistance, CHL: Chloramphenicol, CIP: Ciprofloxacin, ERY: Erythromycin, NIT: Nitrofurantoin, LID: Linezolid, PEN: Penicillin G, TET: Tetracycline, VAN: Vancomycin. 


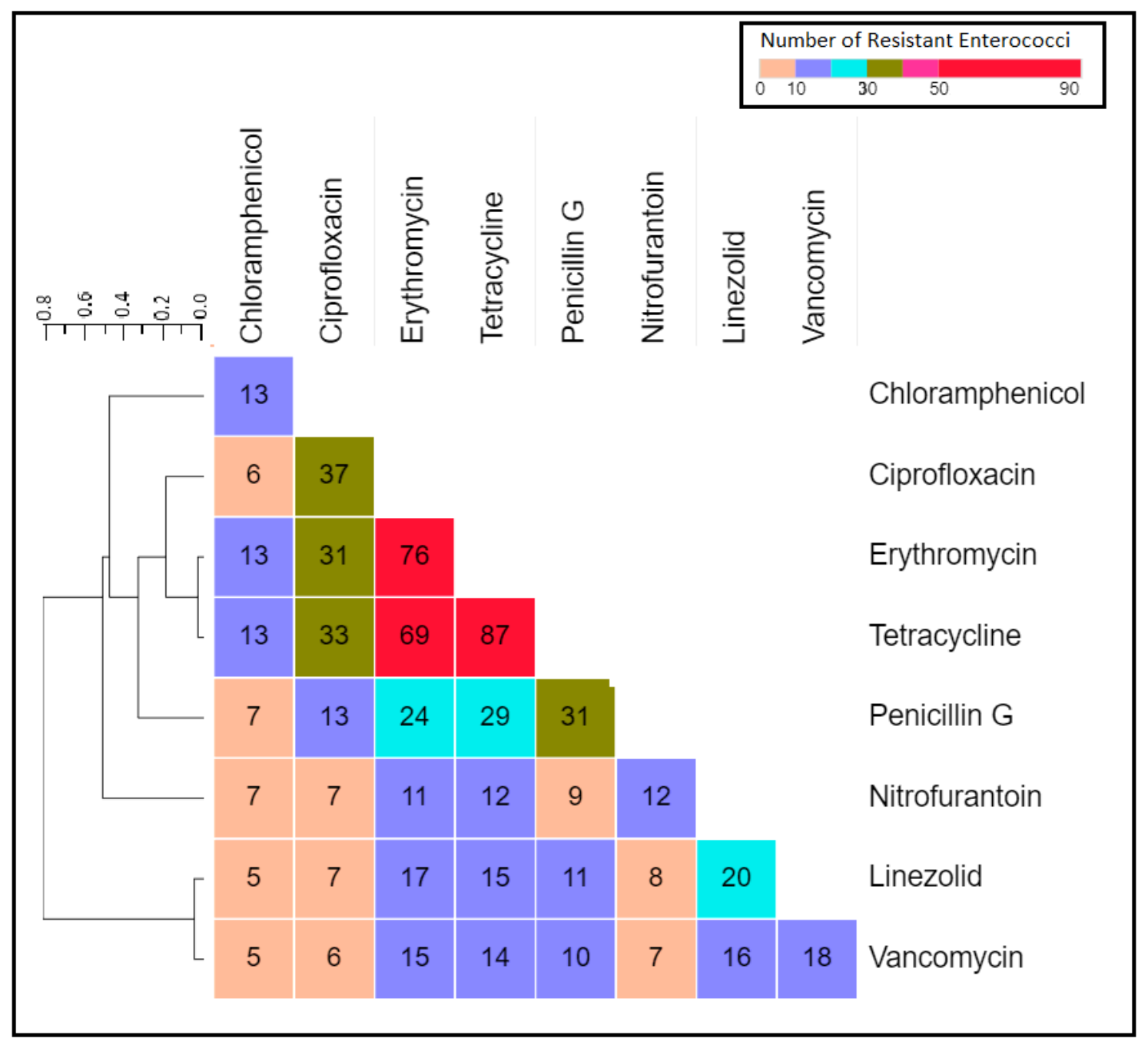

Figure 2

Comparison and dendrogram cluster of pairwise antimicrobial resistance pattern against the all-tested antibiotics: The dual antibiotic resistance pattern was visualized through hit-map similarity matrix and hierarchical clustering was done based on one minus Pearson correlation coefficient and average linkage method. In above matrix the value of each square representing the number of resistant enterococci against both corresponding vertical and horizontal antimicrobials.

\section{Supplementary Files}

This is a list of supplementary files associated with this preprint. Click to download.

- SamadSupplementary02PrevalenceOfEnterococcilnFarmAndMeat.docx

- SamadSupplementary03AMRpatternHostSppeciesGroups.xlsx

- SamadSupplementary04ASTzoneData.xlsx

- SamadSupplementary01ListOfPrimers.docx 